\title{
Numerical Study of Natural Convection of Air in an Inclined Square Enclosure
}

\author{
U. Prasopchingchana, W. Pirompugd, P. Laipradit, and K. Boonlong
}

\begin{abstract}
Natural convection of air in an inclined square enclosure was numerically investigated. The left and right walls of the enclosure were maintained at the uniform hot and cold temperatures, respectively, while the top and bottom walls were adiabatic. The enclosure was filled with real air, a compressible Newtonian fluid. The finite volume method was employed to discretize the partial differential equations of airflow in the enclosure. The angles of the inclination of a square enclosure giving the maximum average Nusselt numbers are $\theta \approx 110^{\circ}$ for $R a$ $=1 \times 10^{3}$ and $\theta \approx 130^{\circ}$ for $3 \times 10^{3} \leq R a \leq 1 \times 10^{4}$.
\end{abstract}

Index Terms-Finite volume method, Inclined square enclosure, natural convection, real air.

\section{INTRODUCTION}

Natural convection is a phenomenon occurred in many engineering applications which the consequence is the flow of air near the surfaces of the solids or liquids such as the airflow in double pane windows, the airflow in double glazing doors of display refrigerators and the airflow in gaps or cavities of building walls. To understand clearly, a lot of researchers have devoted to investigate this phenomenon in order to enhance or reduce this mode of heat transfer.

There is a lot of literature describing about the natural convection phenomenon in the enclosures in the different conditions. De Vahl Davis [1] reported an accurate solution of the equations depicting two-dimensional natural convection in a square cavity with differentially heated side walls. A Boussinesq fluid with Prandlt number 0.71 was employed for the laminar flow in the upright cavity. Furthermore, the laminar and turbulent flow in an upright square cavity was investigated by Barakos, Mitsoulis and Assimacopoulos [2]. Both of a Boussinesq fluid and air which the properties dependent on the temperatures were considered by Agrawal, Ali, Velusamy and Das [3]. They also carried out a correlation for heat transfer during laminar natural convection in an enclosure containing uniform mixture of air and hydrogen. Chen and Chung [4] performed in a different geometry inclined arc-shape cavity filled with the Boussinesq fluid. Al-Farhany and Turan [5] investigated natural convective heat and mass transfer in an inclined rectangular cavity filled with porous medium. The fluid density in the porous medium is described by the Boussinesq

Manuscript received October 19, 2012; revised February 2, 2013.

This research work was supported by The Faculty of Engineering, Burapha University under the research grant No. 27/2554. This support is gratefully acknowledged.

U. Prasopchingchana, W. Pirompugd, P. Laipradit, and K. Boonlong are with Mechanical Engineering Department, Faculty of Engineering, Burapha University,Chonburi, Thailand (e-mail: uthai@ buu.ac.th; worapiro@ eng.buu.ac.th; pramote@ buu.ac.th; kittipong@ buu.ac.th ). approximation. And the other authors [6]-[14] that investigated the natural convection in the cavities in the different conditions also considered the fluids in the cavities to be as the Boussinesq fluid.

From the above review of literature, the study of airflow in an enclosure with differentially heated walls is preliminary for understanding in the natural convection phenomenon. Real air filled in an enclosure which the properties are dependent on the temperatures and pressures has not been numerically investigated. Therefore the aim of this work is to investigate numerically the natural convection of the laminar airflow in an inclined square enclosure filled with real air. In addition, an in-house code was developed for this purpose.

\section{Problem Statement And Mathematical Model}

The geometry of the problem under consideration is shown in Fig. 1. The square enclosure has a width and height of $b$. The angles between the enclosure and the horizontal line are $\theta$. The hot temperature side is designated by $T_{H}$ and the cold temperature side is designated by $T_{C}$. The other sides are well insulated. The enclosure is contained with air.

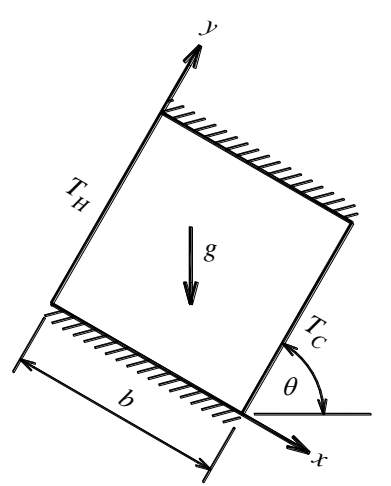

Fig. 1. Geometry of the problem.

Because of the temperature difference between the opposite sides of the enclosure, the air circulation and the natural convection occurs in the enclosure. The governing equations of the airflow in the enclosure are defined by the continuity, momentum and energy equations. The equations at the steady-state condition for a compressible Newtonian fluid are expressed as the following [15]:

- The continuity equation

$$
\nabla \cdot(\rho V)=0
$$

- The momentum equation in $x$ direction

$\nabla \cdot(\rho u V)=-\frac{\partial P}{\partial x}+\nabla \cdot(\mu \nabla u)+\rho g \cos \theta+S_{M x}$ 
where

$$
S_{M x}=\left[\frac{\partial}{\partial x}\left(\mu \frac{\partial u}{\partial x}\right)+\frac{\partial}{\partial y}\left(\mu \frac{\partial v}{\partial x}\right)+\frac{\partial}{\partial x}\left(\left(-\frac{2}{3} \mu\right) \nabla \cdot V\right)\right]
$$

- The momentum equation in $y$ direction

$$
\nabla \cdot(\rho v V)=-\frac{\partial P}{\partial y}+\nabla \cdot(\mu \nabla v)-\rho g \sin \theta+S_{M y}
$$

where

$$
S_{M y}=\left[\frac{\partial}{\partial x}\left(\mu \frac{\partial u}{\partial y}\right)+\frac{\partial}{\partial y}\left(\mu \frac{\partial v}{\partial y}\right)+\frac{\partial}{\partial y}\left(\left(-\frac{2}{3} \mu\right) \nabla \cdot V\right)\right]
$$

- The energy equation

$$
\nabla \cdot(\rho i V)=-P \nabla \cdot V+\nabla \cdot(k \nabla T)+\Phi
$$

where $\Phi=\mu\left\{2\left[\left(\frac{\partial u}{\partial x}\right)^{2}+\left(\frac{\partial v}{\partial y}\right)^{2}\right]+\left(\frac{\partial u}{\partial y}+\frac{\partial v}{\partial x}\right)^{2}\right\}+\left(-\frac{2}{3} \mu\right)(\nabla \cdot V)^{2}$

The appropriate boundary conditions for the airflow in the enclosure are:

$$
\begin{aligned}
\text { - at } x=0,0 & <y<b \\
u & =v=0, T=T_{H}, \frac{\partial P}{\partial x}=0 \\
\text { - at } x=b, 0 & <y<b \\
u & =v=0, T=T_{C}, \frac{\partial P}{\partial x}=0 \\
\text { - at } 0 \leq x \leq b, y & =0 \text { and } y=b \\
u & =v=0, \frac{\partial T}{\partial y}=0, \frac{\partial P}{\partial x}=0
\end{aligned}
$$

All properties of air in the enclosure are functions of the temperatures and pressures. The air properties are obtained from NIST (National Institute of Standards and Technology) Standard Reference Database 23, Version 9.0 by the polynomial curve fitting. The air properties can be obtained from

$$
\begin{aligned}
\phi & =\left(a_{11}+a_{21} T+\ldots+a_{m 1} T^{m-1}\right) \\
& +\left(a_{12}+a_{22} T+\ldots+a_{m 2} T^{m-1}\right) P \\
& +\ldots+\left(a_{1 n}+a_{2 n} T+\ldots+a_{m n} T^{m-1}\right) P^{n-1}
\end{aligned}
$$

where $\phi$ are the variables of density, thermal conductivity, viscosity and specific internal energy, $a_{m n}$ are the polynomial curve fitting coefficients, and $T$ and $P$ are the absolute temperatures and pressures of air in the enclosure, respectively. The coefficients of determination $\left(R^{2}\right)$ of the air properties received from the polynomial curve fitting are more than 0.9990. For all air properties, $m$ and $n$ are 10 .

\section{NUMERICAL METHOD}

The continuity, momentum and energy equations for the simulations of airflow in the enclosure are numerically solved according to their boundary conditions. The finite volume method is employed to discretize the equations of the airflow. The PISO (Pressure Implicit with Splitting of Operators) algorithm is adopted to solve the velocity-pressure coupling problem of the airflow. The hybrid differencing scheme is used to discretize the convective and diffusive terms of the transport equations. The TDMA (Tri-Diagonal Matrix Algorithm) is used to solve the matrix systems. In the numerical procedures, the iteration processes have to be used to obtain the convergent solutions. Consequently, a convergence criterion is established to monitor the maximum relative difference of the velocities, temperatures and pressures in two successive iterations. The maximum relative difference must be less than $10^{-4}$, MAX $\left|\left(\phi^{\text {iter }}-\phi^{\text {iter- } 1}\right) / \phi^{\text {iter }}\right|$ $\leq 10^{-4}$, with $\phi=u, v, T, P$. A summary of the numerical procedures is:

1) The initial guessed values for all of the variables are imposed.

2) The continuity and momentum equations of air in the enclosure are solved under the PISO algorithm to obtain velocity and pressure fields.

3) The energy equations of air in the enclosure are solved to obtain the temperature distribution.

The convergence criterion is applied for all of the variables. If all of the variables do not meet the convergence criterion, it must return to step 2, until the convergence criterion is achieved.

\section{CODE VALIDATION}

The validity of the in-house code is demonstrated by comparing its results with a benchmark numerical solution of De Vahl Davis [1] for natural convection of air in a square cavity with differentially heated vertical walls and adiabatic horizontal walls. The in-house code was run at $\theta=90^{\circ}$ for the comparison.

The Nusselt numbers at any points on the vertical planes in the enclosure excluded thermal radiation is given by

$$
N u=\frac{q_{\text {cond }}+q_{\text {conv }}}{q_{\text {ref }}}
$$

where $q_{\text {cond }}, q_{\text {conv }}$ and $q_{\text {ref }}$ are the conductive, convective and reference heat flux, respectively. The conductive, convective and reference heat flux is calculated as:

$$
\begin{gathered}
q_{\text {cond }}=-k_{A} \frac{\partial T}{\partial x} \\
q_{\text {conv }}=\rho u\left(h+\frac{1}{2}\left(u^{2}+v^{2}\right)\right) \\
q_{\text {ref }}=k_{A} \frac{T_{H}-T_{C}}{b}
\end{gathered}
$$

where $k_{A}$ and $h$ are the thermal conductivity and enthalpy values of air, respectively.

The average Nusselt number on a vertical plane can be written as

$$
N u_{\text {ave }}=\frac{1}{b} \int_{0}^{b} N u d y
$$

The average Nusselt number throughout the enclosure is given by

$$
\overline{N u}=\frac{1}{b} \int_{0}^{b} N u_{a v e} d x
$$


The non-dimensional velocities and coordinates in horizontal and vertical directions are expressed as:

$$
\begin{aligned}
& u^{*}=\frac{u}{\alpha / b}, v^{*}=\frac{v}{\alpha / b} \\
& x^{*}=\frac{x}{b}, y^{*}=\frac{y}{b}
\end{aligned}
$$

The comparisons between the solution of the in-house code and the benchmark numerical solution at $R a=10^{3}$ and $R a=10^{4}$ are shown in TABLE I. The difference between the solution of the in-house code and the benchmark numerical solution is very small, which gives credence to the in-house code.

TABLE I: COMPARISONS BETWEEN THE SOLUTION OF THE IN-HOUSE CODE AND THE BENCHMARK NUMERICAL SOLUTION.

\begin{tabular}{lccc}
\hline \hline & \multicolumn{3}{c}{$R a=10^{3}$} \\
\hline$\overline{N u}$ & 1.118 & 1.118 & 0.000 \\
\cline { 2 - 4 }$N u_{1 / 2, \text { ave }}$ & 1.127 & 1.118 & 0.799 \\
$N u_{0, \text { ave }}$ & 1.117 & 1.117 & 0.000 \\
$N u_{0, \text { max }}$ & 1.503 & 1.505 & 0.133 \\
$\left(0, y^{*}\right)$ & $(0,0.094)$ & $(0,0.092)$ & \\
$N u_{0, \min }$ & 0.693 & 0.692 & 0.144 \\
$\left(0, y^{*}\right)$ & $(0,0.998)$ & $(0,1)$ & \\
$u_{\text {max }}^{*}$ & 3.649 & 3.649 & 0.000 \\
$\left(0.5, y^{*}\right)$ & $(0.5,0.826)$ & $(0.5,0.813)$ & \\
$v_{\text {max }}^{*}$ & 3.702 & 3.697 & 0.135 \\
$\left.x^{*}, 0.5\right)$ & $(0.174,0.5)$ & $(0.178,0.5)$ & \\
\hline
\end{tabular}

\begin{tabular}{lccc}
\hline & \multicolumn{3}{c}{$R a=10^{4}$} \\
\hline$\overline{N u}$ & Present study & De Vahl Davis [1] & Difference (\%) \\
\cline { 2 - 4 }$N u_{1 / 2, \text { ave }}$ & 2.252 & 2.243 & 0.400 \\
$N u_{0, \text { ave }}$ & 2.244 & 2.243 & 1.233 \\
$N u_{0, \max }$ & 3.543 & 2.238 & 0.267 \\
$\left(0, y^{*}\right)$ & $(0,0.142)$ & $(0,0.143)$ & 0.423 \\
$N u_{0, \min }$ & 0.596 & 0.586 & \\
$\left(0, y^{*}\right)$ & $(0,0.998)$ & $(0,1)$ & 1.678 \\
$u^{*}{ }_{\text {max }}{ }^{*}$ & 15.796 & 16.178 & 2.418 \\
$\left.0.5, y^{*}\right)$ & $(0.5,0.826$ & $(0.5,0.823)$ & \\
$v_{\text {max }}^{*}$ & 19.251 & 19.617 & 1.901 \\
$\left.x^{*}, 0.5\right)$ & $(0.116,0.5)$ & $(0.119,0.5)$ & \\
\hline \hline
\end{tabular}

The values in the parentheses are the non-dimensional coordinates.

\section{RESUlTS AND DISCUSSION}

To ensure that the results will not have the deviations because of the grid sizes, The grid independent tests were carried out at $R a=10^{4}, \theta=90^{\circ}$ and the non-uniform grid sizes were $30 \times 30,32 \times 32,34 \times 34,36 \times 36,38 \times 38$, and $40 \times 40$. The grids near the walls of the enclosure are fine while the grids near the central area of the enclosure are coarse. The ratio of the width of the grids near the central area of the enclosure to the width of the sequential grids near the walls of the enclosure is 1.2. The average Nusselt number throughout the enclosure, $\overline{N u}$, was adopted to be the criteria test value. The change of $\overline{N u}$ is less than 0.01 percent, when the grid sizes are larger than or equal $36 \times 36$. Therefore, the non-uniform grid size $38 \times 38$ was employed throughout this work. The dimensions of the enclosure are $20 \times 20 \mathrm{~mm}$.

Fig. 2. and 3. present the local Nusselt numbers on the hot and cold walls for the different angles of the inclination of the enclosure at $R a=10^{3}$ and $10^{4}$. The local Nusselt numbers on the hot wall near the bottom of the enclosure have the large valves because of the steep temperature gradients shown in Fig. 5., and the values decrease along the distance in the $y$ direction of the hot wall of the enclosure, while the local Nusselt numbers on the cold wall of the enclosure have the values in the different way.

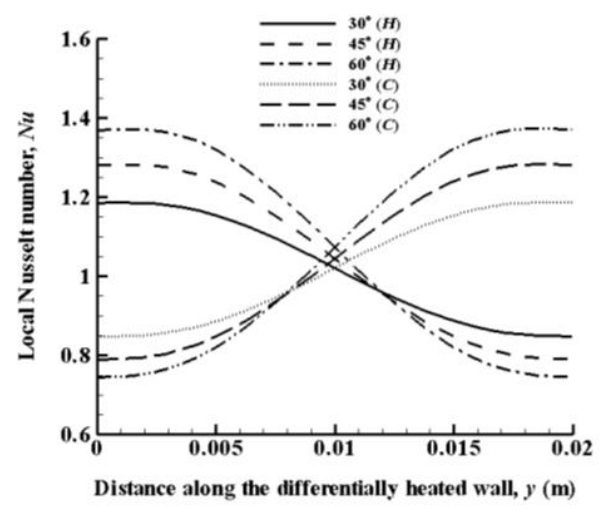

(a) $\theta=30^{\circ}, 45^{\circ}$ and $60^{\circ}$

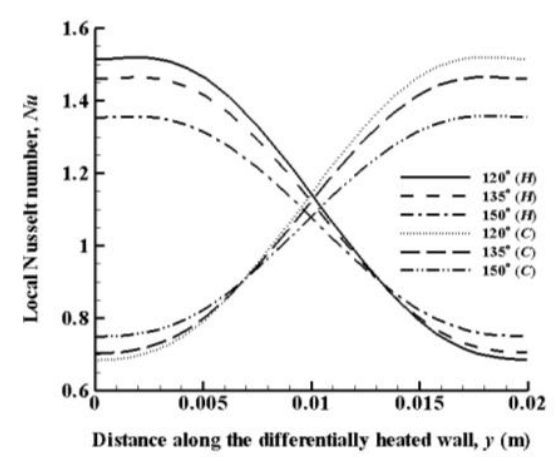

(a) $\theta=120^{\circ}, 135^{\circ}$ and $150^{\circ}$

Fig. 2. Comparison between the local Nusselt numbers on the hot and cold walls for different angles of inclination $\theta$ at $R a=10^{3}$.

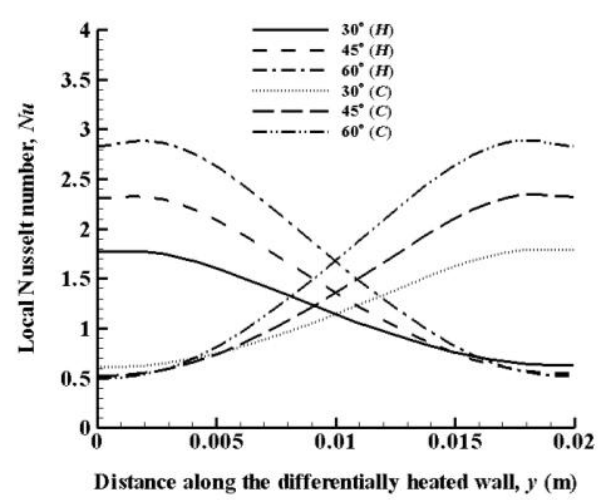

(a) $\theta=30^{\circ}, 45^{\circ}$ and $60^{\circ}$ 


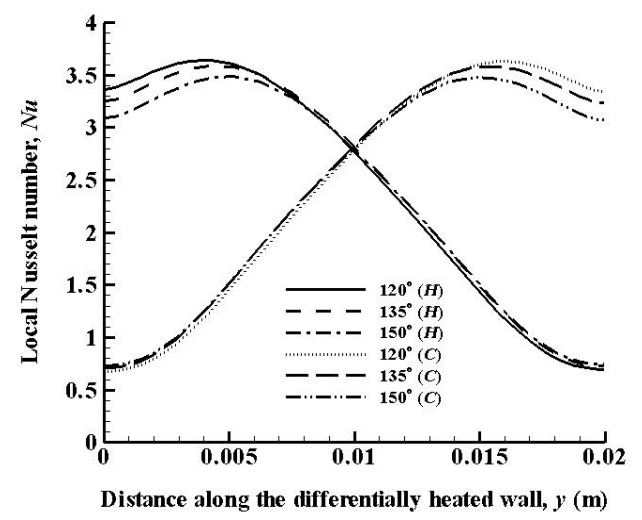

(a) $\theta=120^{\circ}, 135^{\circ}$ and $150^{\circ}$

Fig. 3. Comparison between the local Nusselt numbers on the hot and cold walls for different angles of inclination $\theta$ at $R a=10^{4}$.

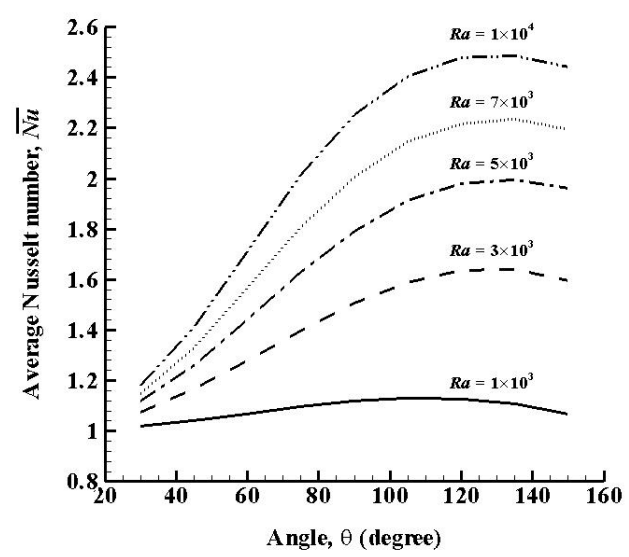

Fig. 4. The average Nusselt numbers for different angles of inclination $\theta$.

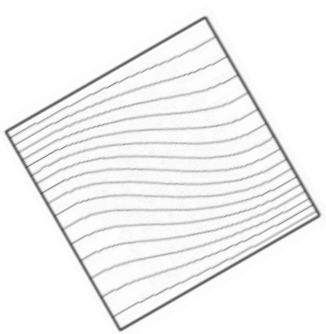

(a) $\theta=30^{\circ}$

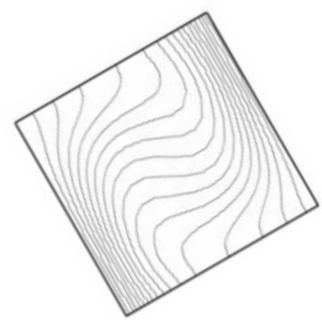

(c) $\theta=120^{\circ}$

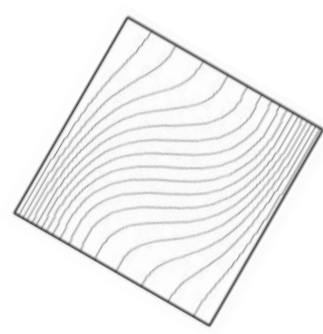

(b) $\theta=60^{\circ}$

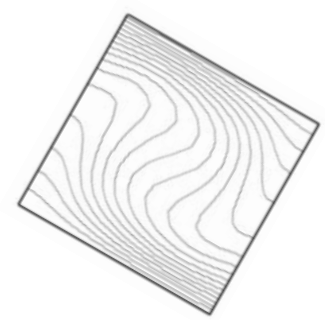

(d) $\theta=150^{\circ}$
Fig. 5. Temperature contours of air in an inclined square enclosure for different angles of inclination $\theta$ at $R a=10^{4}$.

Fig. 4. shows the average Nusselt numbers for the different angles of the inclination of the enclosure at $R a=1 \times 10^{3}$, $5 \times 10^{3}, 7 \times 10^{3}$ and $1 \times 10^{4}$. The average Nusselt number values vary according to the Rayleigh number values and the maximum average Nusselt numbers occur at $\theta \approx 110^{\circ}$ for $R a=$ $1 \times 10^{3}$ and $\theta \approx 130^{\circ}$ for $3 \times 10^{3} \leq R a \leq 1 \times 10^{4}$.
Fig. 6. shows the velocity vectors of the airflow in the inclined square enclosure at $R a=10^{4}$. The velocity vectors of the airflow have larger values when the hot wall of the enclosure faces upward because hot air is easy to flow up to the cold wall of the enclosure in this condition.

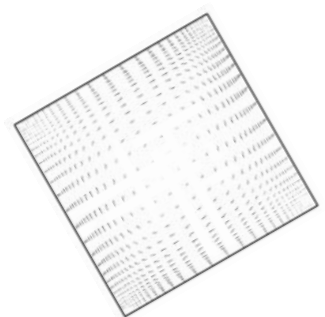

(a) $\theta=30^{\circ}$

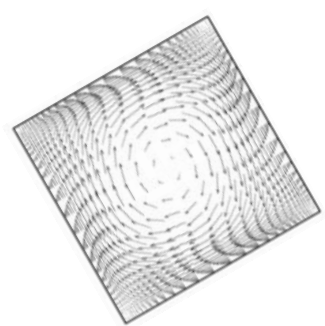

(c) $\theta=120^{\circ}$

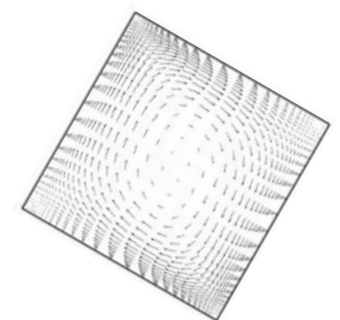

(b) $\theta=60^{\circ}$

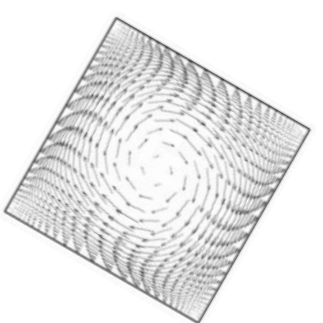

(d) $\theta=150^{\circ}$
Fig. 6. Velocity vectors of airflow in an inclined square enclosure for different angles of inclination $\theta$ at $R a=10^{4}$.

\section{CONCLUSION}

\section{NOMENCLATURE}

\begin{tabular}{|ll|}
\hline$g$ & gravitational acceleration, $\mathrm{m} / \mathrm{s}^{2}$ \\
$h$ & enthalpy, $\mathrm{J} / \mathrm{kg}$ \\
$k$ & thermal conductivity, $\mathrm{W} / \mathrm{m} \cdot \mathrm{K}$ \\
$N u$ & Nusselt number \\
$P$ & pressure, $\mathrm{Pa}$ \\
$R a$ & Rayleigh number \\
$T$ & temperature, $\mathrm{K}$ \\
$u$ & velocity component in $x$ direction, $\mathrm{m} / \mathrm{s}$ \\
$v$ & velocity component in $y$ direction, $\mathrm{m} / \mathrm{s}$ \\
$V$ & total velocity, $\mathrm{m} / \mathrm{s}$ \\
$x$ & Cartesian coordinate in high direction of the \\
& enclosure, $\mathrm{m}$ \\
$y$ & Cartesian coordinate in wide direction of the \\
Greek symbols & enclosure, $\mathrm{m}$ \\
$\alpha$ & thermal diffusivity, $\mathrm{m}^{2} / \mathrm{s}$ \\
$\theta$ & inclination angle of the enclosure, \\
$\mu$ & viscosity, $\mathrm{kg} / \mathrm{s} \cdot \mathrm{m}$ \\
$\rho$ & density, $\mathrm{kg} / \mathrm{m}^{3}$ \\
Mathematical symbol \\
$\nabla$ & del operator \\
Superscript \\
iter & iteration number \\
Subscripts & air \\
A & cold wall \\
$H$ & hot wall \\
\hline
\end{tabular}

For the natural convection occurring in the square 
differentially heated wall enclosure, the average Nusselt numbers have larger values when the Rayleigh numbers have larger values. The local Nusselt numbers on the hot and cold walls of the enclosure will have larger values when those areas have the steeper temperature gradients. The angles of the inclination of the enclosure giving the maximum average Nusselt numbers are $\theta \approx 110^{\circ}$ for $R a=1 \times 10^{3}$ and $\theta \approx 130^{\circ}$ for $3 \times 10^{3} \leq R a \leq 1 \times 10^{4}$.

\section{ACKNOWLEDGMENT}

This research work was supported by The Faculty of Engineering, Burapha University under the research grant No. 27/2554. This support is gratefully acknowledged.

\section{REFERENCES}

[1] G. D. V. Davis, "Natural convection of air in a square cavity a bench mark numerical solution," International Journal for Numerical Methods in Fluids, vol. 3, pp. 249-264, 1983.

[2] G. Barakos, E. Mitsoulis, and D. Assimacopoulos, "Natural convection flow in a square cavity revisited: laminar and turbulent models with wall functions," International Journal for Numerical Methods in Fluids, vol. 18, pp. 695-719, 1994.

[3] N. Agrawal, S. M. Ali, K. Velusamy, and S. K. Das, "A correlation for heat transfer during laminar natural convection in an enclosure containing uniform mixture of air and hydrogen," International Communications in Heat and Mass Transfer, vol. 39, pp. 24-29, 2011.

[4] C. L. Chen and Y. C. Chung, "Parametric study on mixed convection heat transfer in an inclined arc-shape cavity," International Communications in Heat and Mass Transfer, vol. 39, pp. 610-616, 2012.

[5] K. A. Farhany and A. Turan, "Numerical study of double diffusive natural convective heat transfer in an inclined rectangular cavity filled with porous medium," International Communications in Heat and Mass Transfer, vol. 39, pp. 174-181, 2011.

[6] M. Mahmoodi, "Numerical simulation of free convection of nanofluid in a square cavity with an inside heater," International Journal of Thermal Sciences, vol. 50, pp. 2161-2175, 2011.

[7] A. K. A. Hakeem, S. Saravannan, and P. Kandaswamy, "Natural convection in a square cavity due to thermally active plates for different boundary conditions," Computers and Mathematics with Applications, vol. 62, pp. 491-496, 2011

[8] G. Yesiloz and O. Aydin, "Natural convection an inclined quadrantal cavity heated and cooled on adjacent walls," Experimental Thermal and Fluid Sciences, vol. 35, pp. 1169-1176, 2011.

[9] E. J. Braga and M. J. S. de Lemos, "Laminar and turbulent free convection in a composite enclosure," International Communications in Heat and Mass Transfer, vol. 52, pp. 588-596, 2008.

[10] P. Kandaswamy, J. Lee, A. K. A. Hakeem, and S. Saravanan, "Effect of buffle-cavity ratios on buoyancy convection in a cavity with mutually orthogonal heated baffles," International Journal of Heat and Mass Transfer, vol. 51, pp. 1830-1837, 2008.

[11] A. K. Sharma, K. Velusamy, C. Balsji, and S. P. Venkateshan, "Conjugate Turbulent natural convection with surface radiation in air filled rectangular enclosures," International Journal of Heat and Mass Transfer, vol. 50, pp. 625-639, 2007.

[12] B. Calcagni, F. Marsili, and M. Paroncini, "Natural convective heat transfer in square enclosures heared from below," Applied Thermal Engineering, vol. 25, pp. 2522-2531, 2005.

[13] O. Aydin, A. Unal, and T. Ayhan, "Natural convection in rectangular enclouses heated from one side and cooled from the ceiling," International Journal of Heat and Mass Transfer, vol. 42, pp. 2345-2355, 1999.

[14] A. Bairi, "Nusselt-Rayleigh correlations for design of industrial elements: Experimental and numerical investigation of natural convection in tilted square air filled enclosures," Energy Conversion \& Management, vol. 49, pp. 771-782, 2008.

[15] H. K. Versteeg and W. Malalasekera, An introduction to Computational Fluid Dynamics The finite volume Method, $2^{\text {nd }}$ ed. Essex, England: Pearson Education Limited, 2007.

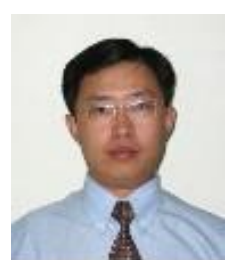

Uthai Prasopchingchana received his B.Eng. and M.Eng. in Mechanical Engineering at Chularlongkorn University, Thailand. He then completed M.S. in Mechanical Engineering at Drexel University, U.S.A. in 2008. He has been working in Mechanical Engineering Department, Faculty of Engineering, Burapha University, Thailand for thirteen years. His research works are focused on Heat Transfer and Computational Fluid Dynamics.

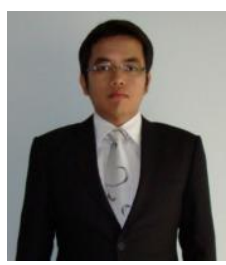

Worachest Pirompugd received his B.Eng., M.Eng., and D.Eng. in Mechanical Engineering at King Mongkut's University of Technology Thonburi, Thailand. He has been working in Mechanical Engineering Department, Faculty of Engineering, Burapha University, Thailand for over twelve years. His research works are focused on Heat and Mass Transfer in Fin-and-Tube Heat Exchangers and Two Phase Flow in Capillary Tubes.

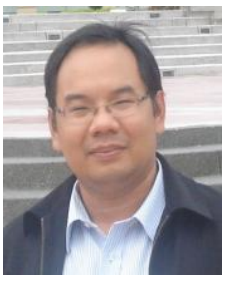

Pramote Laipradit received his B.Eng. in Mechanical Engineering at Chiang Mai University, Thailand. He then completed M.Eng. in Energy Technology and Ph.D. in Thermal Technology at King Mongkut's University of Technology Thonburi, Thailand. He has been working in Department of Mechanical Engineering, Faculty of Engineering, Burapha University, Thailand for six years. His research works are focused on Refrigeration and Heat Pumps.

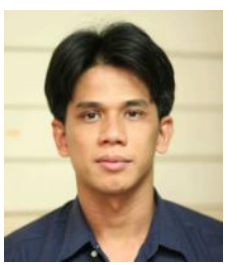

Kittipong Boonlong received B.Eng. from Prince of Songkla University, Thailand in 1998, M.Eng. from King Mongkut's University of Technology North Bangkok, Thailand in 2001, and Ph.D. from Chulalongkorn University, Thailand in 2005. He is currently an assistant professor in Mechanical Engineering, Faculty of Engineering, Burapha University, Thailand. His main research interests include Single and Multiple Objective Optimization, Topology Optimization, Evolutionary Algorithms, Mechanical Vibration, Damage Detection, Finite Volume and Finite Element Methods. 